\title{
Ventral tethering in split cord malformation
}

\author{
Dachling Pang, M.D., F.R.C.S.(C), F.A.C.S. \\ Department of Neurological Surgery, Kaiser Permanente Hospitals; and Department of \\ Neurosurgery, University of California at Davis, Sacramento, California
}

\begin{abstract}
Object. The author studied the specific issue of ventral tethering in cases of type II split cord malformation (SCM). He also discussed the issue of ventral tethering in patients with type I SCM in whom a bony septum and dural sleeve have been resected.

Methods. Eleven (21\%) of 52 patients with type II SCM were found to have a ventral fibrous septum tethering the hemicords to the ventral dura. Four categories of ventral septa were noted: 1) pure ventral intradural septa (three patients); 2) complete dorsoventral intradural septa (three patients); 3) dorsoventral septa continuous with a dermal sinus tract (two patients); and 4) ventral or complete septa continuous with ventral intestinal bands causing intestinal malrotation or diverticulum (three patients).

Excluding one autopsy case, all 10 patients had symptoms or progressive neurological syndromes at diagnosis. Magnetic resonance imaging was completely ineffective, and computerized tomography (CT) myelography was only partially effective in detecting ventral tethering in 50\% of cases. The only other positive predictor of ventral tethering is the association of dermal sinus tract and intestinal malformations. Combining this predictor with CT myelography, only $60 \%$ of the cases of ventral tethering could be suspected before surgery.

Conclusions. The author recommends examining all patients with type II SCM, and at surgery the ventral surface of the split length and reunion site should be thoroughly inspected to reveal a ventral band.
\end{abstract}

\section{KEY WORDS - split cord malformation • ventral tethering • median fibrous septum • embryogenesis • intestinal malformation • dermal sinus}

It is now well accepted that all double spinal cord malformations, or SCM, probably arise from a common embryogenetic mechanism. ${ }^{25}$ Pang and coworkers ${ }^{23,25}$ have postulated that the participation of certain meningeal precursor cells (cells from primitive meninx) within an errant midline ectoendodermal adhesion tract during early gastrulation determines how SCMs can be classified into two radically different types. Type I SCM consists of two hemicords contained within separate dural tubes, separated by a bone or osteocartilaginous septum that extends from the VB to the neural arches. This rigid median septum is therefore entirely extradural. In type II SCM, the hemicords reside within a common dural tube. There is no rigid median septum. These main features of the two types of SCM never overlap, ${ }^{25}$ and the classification can be readily made by preoperative neuroimaging studies. ${ }^{23}$

Even before the publication of the unified theory of embryogenesis of SCMs, type I SCMs (previously termed diastematomyelia) were known to be serious tethering lesions. The transfixing, bladelike bone septum was never regarded as anything but deleterious to the hemicords, and the necessity of its removal was seldom disputed. On the other hand, it took some convincing to show that the hemicords in many cases of type II SCM were also se-

Abbreviations used in this paper: CSF = cerebrospinal fluid; $\mathrm{CT}=$ computerized tomography; $\mathrm{MR}=$ magnetic resonance; $\mathrm{SCM}=$ split cord malformation; $\mathrm{VB}=$ vertebral body . verely tethered by taut fibrous bands and septa that are attached to the common dural sac. ${ }^{23}$ Many of the patients with these lesions deteriorate neurologically and recover promptly after resection of the fibrous septum. The results of other series have since upheld these views. ${ }^{1,9,26}$ Thus, despite the fact that MR imaging and CT myelography often do not demonstrate these thin but meddlesome fibrous septa, the author and others have recommended the surgical exploration of all type II SCMs. ${ }^{9,23}$

The aforementioned recommendation was made in 1992 with the reassurance that exploring a type II SCM was technically easy and safe, because there was no interfering bone septum and that excision of a few uncomplicated dorsal fibrous bands and sheaths was all that was necessary to untether the hemicords. ${ }^{23,24}$ However, in the same series the author also reported several examples of type II lesion in which the fibrous band was hidden on the ventral side of the hemicords, ${ }^{22}$ and he cautioned that eliminating these ventral bands involved a slightly more extensive surgery and noted that detecting them required a heightened vigilance.

Considering the original endomesenchymal tract began as a median structure conjoining the endoderm and ectoderm, traversing all the elements that later form the spinal canal, it is hardly surprising to find that these mesenchyme-derived fibrous bands sometimes retain ventral attachment to the hemicords. Indeed, as my experience with SCM grows, I have found more examples of purely ventral intradural bands in type II SCMs, as well as dor- 
soventral bands that bisect the entire sagittal plane of the dura. There are even cases in which the ventral mesenchymal band extends beyond the vertebral column to the extraluminal tissues of the gut (endoderm), resulting in characteristic gastrointestinal malformations. The specific issue of ventral tethering in type II SCMs is addressed in this paper.

In addition, I have also encountered patients with type I SCMs in whom ventral tethering develops following the resection of the bone septum and its dural sleeve. This kind of acquired ventral tethering in type I lesions is always due to ill-advised techniques and can be avoided. This aspect of ventral tethering in type I SCM will also be discussed.

\section{CLINICAL MATERIAL AND METHODS}

\section{Patient Population}

The clinical materials for this study is culled from a series of 97 patients with SCM treated by the author between January 1979 and December 1999, mainly at three institutions (the University of Pittsburgh, University of California at Davis, and Kaiser Permanente Hospitals of Northern California). A few patients were treated at overseas universities while the author was a visiting professor.

Of this group, a simple type I SCM was diagnosed in 44 patients; a simple type II SCM in 47 patients; and composite SCMs consisting of more than one SCM were documented in six patients. Of the six cases of composite SCM, one patient harbored two noncontiguous type I lesions, two harbored a contiguous arrangement of two type I lesions sandwiching a type II lesion, and three had double SCMs, each consisting of a type I and a type II lesion separated by variable lengths of normal spinal cord. Thus, altogether there were 52 type II SCMs.

Fifty-one of the 52 type II lesions were surgically explored. One infant died at 3 days of life following surgery for a diaphragmatic hernia, and a detailed autopsy examination revealed the type II SCM. Of these, 11 cases had definite ventral tethering of the hemicords. This subgroup of 11 cases makes up the bulk material of the current study. There were one adult and 10 children ranging from birth to 18 years of age. The female/male ratio was 7:4.

\section{Reasons Leading to Diagnosis}

In this subgroup, two type II SCMs were found on routine MR imaging screening following closure of a myelomeningocele but, retrospectively, it was determined that both children had symptoms of tethering. In seven other children and one adult, the SCM was found during investigation into the causes of progressive neurological deterioration. The one autopsy case previously mentioned makes up the 11th case in the series.

\section{Neuroimaging Evaluation}

Computerized tomography myelography (with metrizamide or iohexol) was performed in all patients except for the autopsy case. In some patients, the approximate location of the split segment was revealed on MR imaging so that detailed axial CT cuts could be directed accordingly. In all cases, nonoverlapping 1.5 or $3.0-\mathrm{mm}$ axial sections obtained using bone algorithm were used to define details of the entire split segment, the conus medullaris, and any other suspicious area along the spinal axis such as a second split cord. A bone algorithm was used because it best displayed thin interruption in the contrast column that might represent a fibrous septum, and it provided the most credible evaluation of the anterior bifid condition of the VB.

Nine patients underwent MR imaging. The sensitivity of MR imaging in defining the ventral tethering was compared with that of CT myelography.

\section{Surgical Treatment}

In accordance with our treatment protocol for type II SCMs,${ }^{22,24}$ all patients in this subgroup underwent surgical exploration, except for the autopsy case. The aim at surgery was to remove all elements that tethered the hemicords, either directly at the split site or immediately adjacent to it. In every case since our discovery of the first ventral septum early in the series, the ventral aspect of the split region was thoroughly inspected, either by gently rotating the hemicords upward and to one side or through the median cleft. Four aspects of the ventral tethering are documented: whether the septum is 1) a purely ventral septum without dorsal attachment to the hemicords; 2) a complete dorsoventral sheath bisecting the entire dural sac; 3 ) a complete septum continuous with a midline dorsal extradural band or tract that extends to the skin as a dermal sinus opening; or 4) a ventral intradural band continuous with some type of extradural ventral tract attached to the primitive gut. Cases associated with the fourth category were invariably associated with gastrointestinal malformations.

All other tethering lesions except the median fibrous septum were also treated. In all cases of myelomeningocele manqués composed of blood vessels, dorsal paramedian nerve roots, and fibrous bands, the myelomeningocele was cut flush with the surface of the hemicords. All thickened terminal filum were divided.

\section{RESULTS}

\section{Types of Ventral Tethering}

There are essentially four categories of ventral tethering in type II SCM.

Purely Ventral Fibrous Septum. Three patients had a purely ventral fibrous septum (Fig. 1 upper). The hemicords were unattached to the dorsal dura. Each ventral septum arose from the midline ventral dura and was tightly adherent to the caudal reunion point of the hemicords at variable distance rostral to the point of origin on the dura (Fig. 1 center and lower). The septum was thus obliquely angled rostrally so that lifting up the hemicords before the reunion site might not reveal the septum unless the reunited cord below was also gently lifted and rotated away from the dura. In a transcleft exploration the lesion would surely be missed. The septum was often edged by a small artery.

Complete Intradural Dorsoventral Septum. Three patients harbored through-and-through fibrous septa or thick bands that were attached to both the ventral and dorsal dura. The hemicords were thus doubly tethered on both sides. In one case, the ascension of the neural tube along 


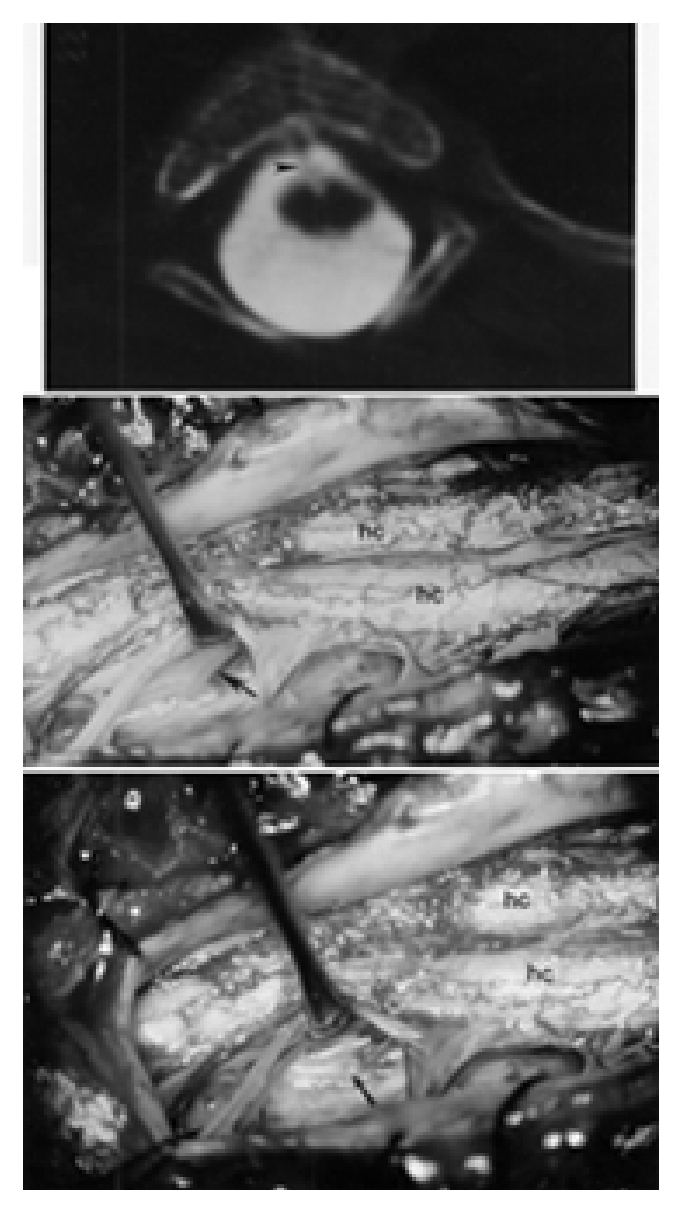

Fig. 1. Type II SCM with a purely ventral fibrous septum. Upper: A CT myelogram obtained at the hemicord reunion site demonstrating the ventral septum (arrowhead). Center: At operation, the cord just caudal to the hemicord (hc) reunion is lifted to reveal the purely ventral, midline fibrous septum with a vessel skirting its anterior edge (arrow). Note that the direction of the septum is from caudal to rostral, pointing exactly at the caudal-most extent of the split cord. Lower: After excision of the ventral septum, its point of entry (arrow) through the midline ventral dura marks the site of the original endomesenchymal tract.

the vertebral/dural column after formation of the endomesenchymal tract presumably carried the attachment of the septum rostrally, resulting in an arrowhead-shaped septum pointing cephalad.

In two children, the complete fibrous septum was oblique from side to side, and it separated a ventral wellformed hemicord from a dorsally located hemimyelomeningocele. The dorsal hemineural plate never neurulated and was opened to the cutaneous defect in a manner identical to the common variety of open neural tube defect. In fact, at the initial closure of the open defect, the hemineural plate was thought to be the whole placode, the other well-neurulated hemicord being shielded from view by the complete fibrous septum, which in turn was mistaken for the ventral dura (Fig. 2). ${ }^{22,25}$

Complete Dorsoventral Septum Continuous With a Dermal Sinus Tract. Figure 3 upper left provides an illustration of complete dorsoventral septum continuous with a dermal sinus tract.

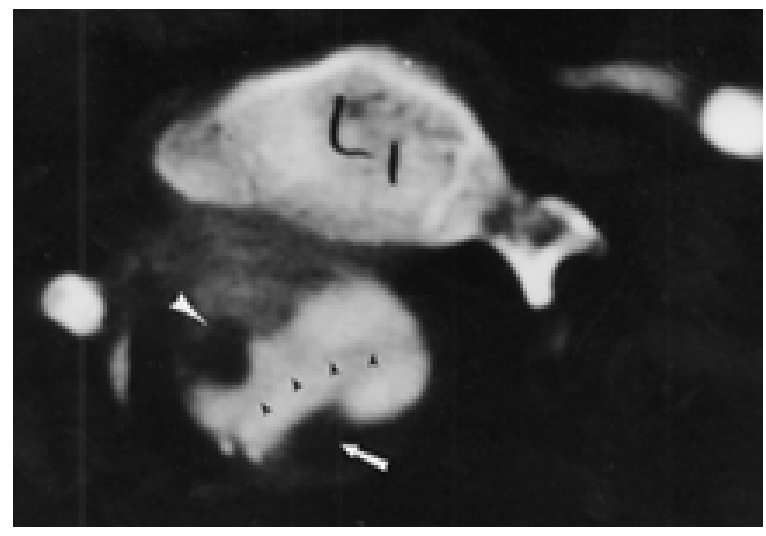

Fig. 2. Computerized tomography myelography revealing a complete dorsoventral fibrous septum in a hemimyelomeningocele. The oblique septum (black arrowheads) separates the dorsal hemineural placode (white arrow), which is adherent to the dorsal dura, from the ventral (neurulated) hemicord (white arrowhead) shielded from view at the original operation.

In two patients, the complete intradural fibrous septum was continuous with an extradural dermal sinus tract that opened onto an obvious cutaneous dermal sinus (Fig. 3 upper right). In one case, the sinus tract expanded into a large intradural dermoid cyst (Fig. 3 lower left). The deep stem of the cyst was continuous with the midline fibrous septum (Fig. 3 lower right), which in turn was followed ventrally through a tidy round opening into the VB, together with bunches of blood vessels, nerves, and jagged fibrous bands.

Ventral Septum Attached to the Primitive Gut. In three patients the ventral intradural septum was continuous with remnants of the ventral endomesenchymal tract that retained attachment to the primitive gut, resulting in rather exotic intestinal malformations. One infant died of severe bronchopulmonary hypoplasia 3 days after transthoracic resection of an intestinal diverticulum (duplication) that presented as diaphragmatic eventration. At autopsy, the mediastinal stump of a stout fibrous tract, distally connected to the apex of the resected ileal diverticulum, was painstakingly traced to a bifid C-7 VB. The tract penetrated the bifid gap, ran through a smooth anterior dural opening, and attached to the ventral midline of a deeply cleft spinal cord (Fig. 4 left). Within this tract were embedded muscles, nerves, and glial tissue (Fig. 4 right).

In two children, the ventral septa were associated with intestinal malrotation. In one child, a complete dorsoventral septum at C3-4 (Fig. 5 upper left, center, and right) was traceable through a bifid C-4 VB to a prevertebral neurenteric cyst (Fig. 5 lower left), which was followed radiographically to the lower mediastinum. Here a fibrous band (a Ladd band) strapped across the fourth part of the duodenum to reach the ileocecal junction causing partial bowel obstruction (Fig. 5 lower center) This band was also responsible for the complete intestinal malrotation in this patient (Fig 5 lower right). ${ }^{25}$ In another child with malrotation, the tethering band was purely ventral and was at the midthoracic level.

A summary of the categories of ventral tethering is depicted in Fig. 6. 


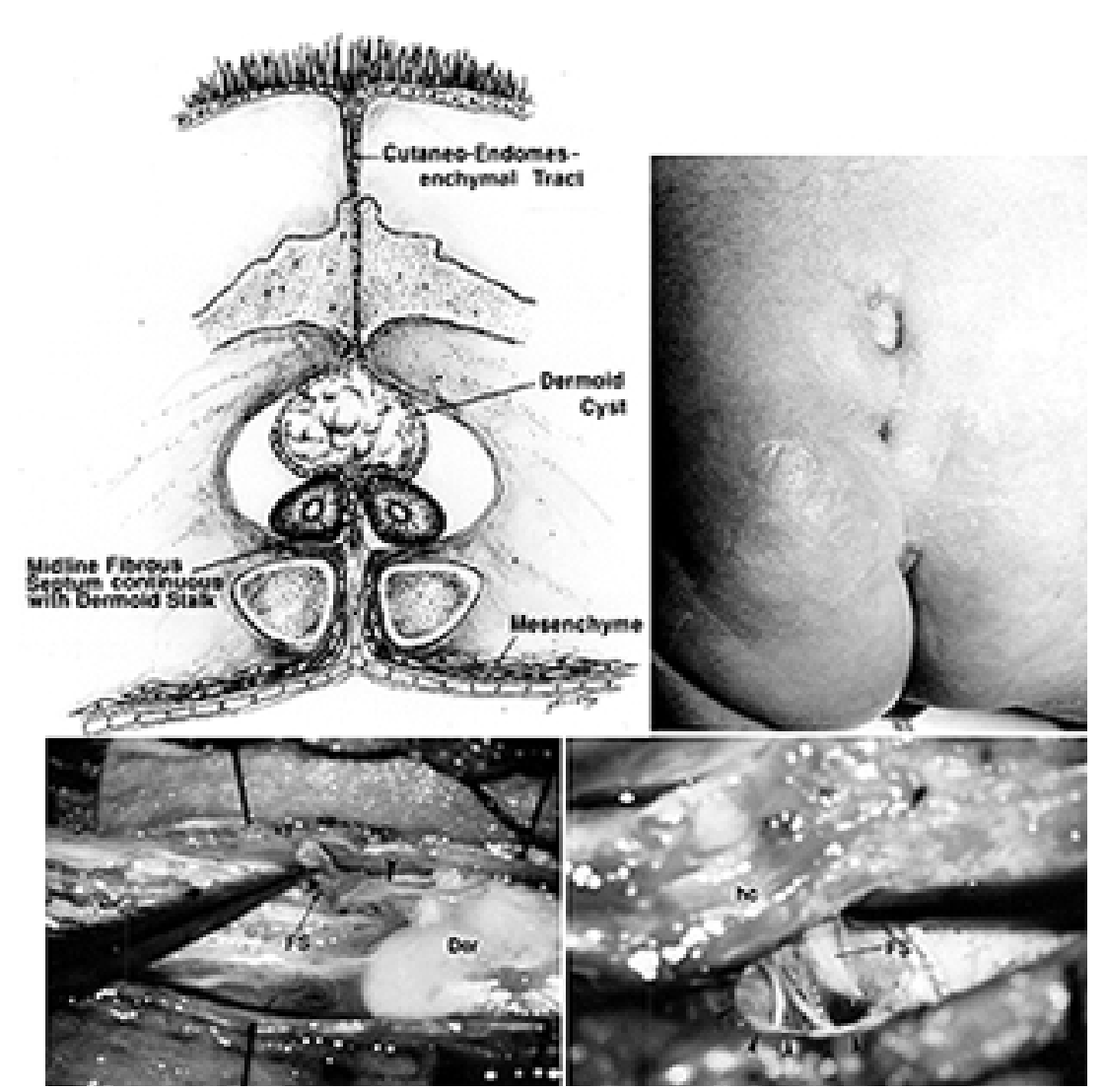

Fig. 3. Complete dorsoventral fibrous septum in a type II SCM associated with a dermal sinus tract. Upper Left: Drawing showing the cutaneoendomesenchymal tract and the dermoid cyst continuous with the median fibrous septum that penetrates the ventral dura. Upper Right: Photograph of a dermal sinus opening that marks the dorsal extreme of this tract. Lower Left: At operation, the caudal stem (arrowhead) of the dermoid cyst (Der) is continuous with the dorsal fibrous septum (FS), held by forceps. Lower Right: After the hemicords are lifted, the ventral fibrous septum, together with nerves and blood vessels, are seen to go through a ventral defect (outlined by arrowheads) in the dura.

\section{Location of the Ventral Septum}

The location of the ventral septa are shown in Fig. 7. There were two cervical, three thoracic, and six lumbosacral septa. The overall distribution was not unlike that of other type II SCM. ${ }^{23}$

\section{Location of the Conus Medullaris}

As with the other type II SCMs ${ }^{23}$ the two cervical ventral septa were associated with normal location of the conus medullaris - that is, at or above the lower border of the L-1 vertebra. Neither patient had an abnormal terminal filum. The thoracic and lumbosacral septa were all associated with a low-lying conus medullaris additionally tethered by a thick filum.

\section{Myelomeningocele Manqué}

Myelomeningocele manqués were found in six patients, either directly from the dorsal aspects of the hemicords or from the adjacent reunited cord. There were multiple sets of fibroneurovascular bands in each case. Four were entangled with the dorsal portion of a through-and-through septum; two sets were found on hemicords without a dorsal septum.

Myelomeningocele manqués were never found on the ventral side of the hemicords.

\section{Neurological Status}

Excluding the infant who died, 10 patients were available for neurological assessment at diagnosis (Fig. 8).

All 10 patients suffered neurological deterioration and/ or pain. Six patients (two thoracic and four with lumbosacral septa) experienced severe spinal pain. In four of these dysesthetic pain was also present in the legs. In six patients (one with cervical, two with thoracic, and three with lumbar septa) signs of progressive myelopathy were documented. One patient with cervical SCM also had clumsy hands. All six patients with lumbosacral lesions suffered adverse changes in their bladder and urodynamic data, either developing more unstable detrusor contractions and higher intravesicular pressure, or frank detrusor-sphincter dyssynergia. In two patients with thoracic disease worsening scoliosis developed.

Both of the children with intestinal malrotation developed small-bowel obstruction during the course of the evaluation.

\section{Neuroimaging Studies}

Excluding the autopsy case, 10 patients underwent neuroimaging studies.

None of the nine MR imaging studies revealed even the slightest hint of a fibrous septum. 


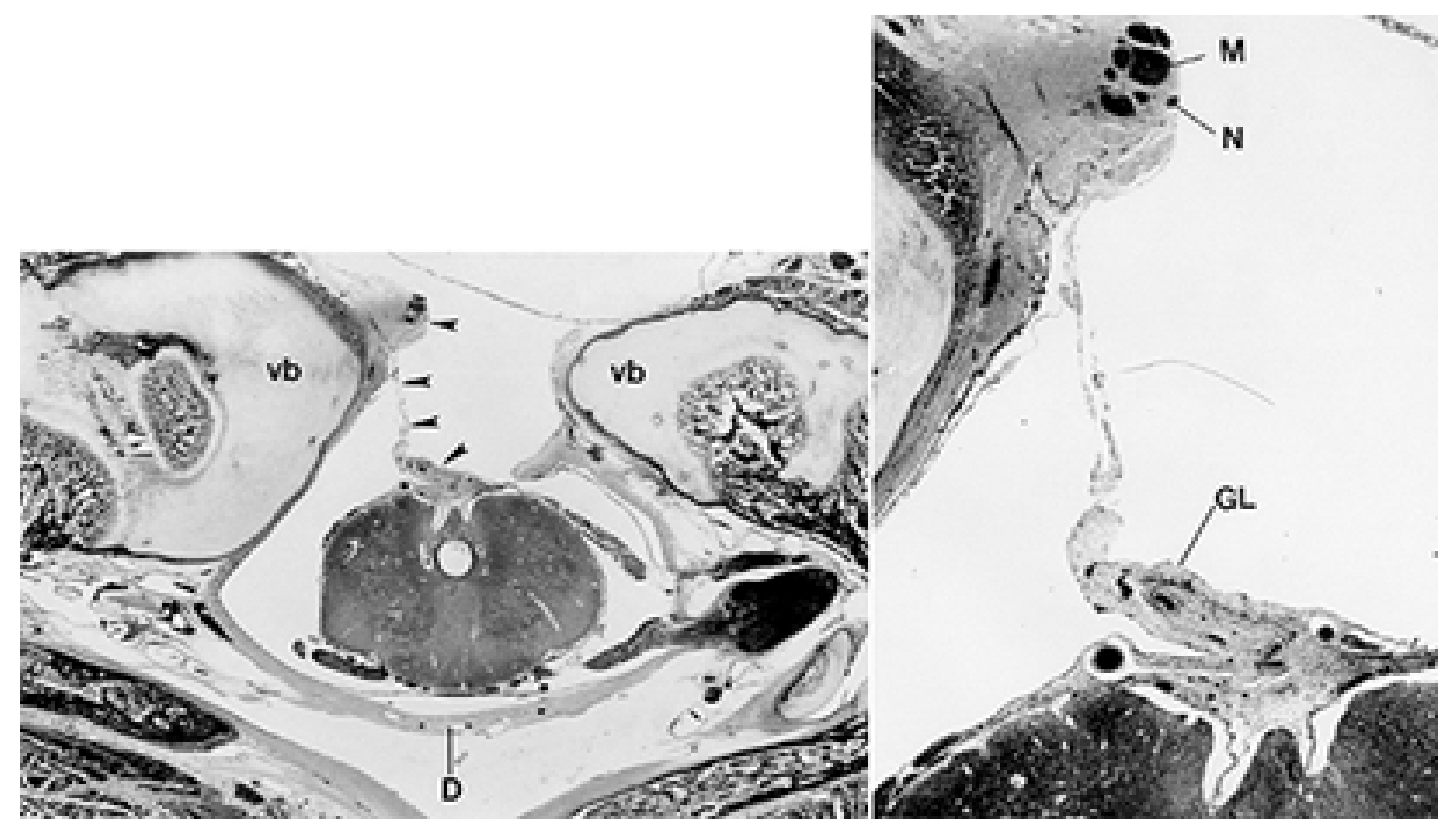

Fig. 4. Ventral fibrous septum associated with intestinal duplication (diverticulum) in a 3-day-old infant who died after surgery for diaphragmatic hernia. Left: Low-power photomicrograph obtained through the C-7 vertebra showing the completely bifid VB and anterior dural defect. The fibrous tract (arrowheads) is attached to the anterior midline of a deeply cleft cord and goes through the anterior dural defect near the left bank of the bifid VB. D = dura. Masson trichrome, original magnification $\times 40$. Right: High-power photomicrograph showing that the fibrous tract is embedded with glial tissue $(\mathrm{GL})$, nerves $(\mathrm{N})$, and muscles $(\mathrm{M})$, original magnification $\times 80$.

Of the 10 CT myelography studies obtained, three revealed a definite ventral septum (Fig. 1 upper), two of which were through-and-through dorsoventral bands, including one with a hemimyelomeningocele (Fig. 2). In two studies, a ventral midline "something" was suspected but not a definite septum (Fig. 9). No midline septum of any kind was revealed in four CT myelography studies. In the case of a large intradural dermoid cyst, the dorsal portion of a very thick fibrous septum was well demonstrated on CT myelography studies but not the ventral portion of the sheath (Fig. 10). In all, three ventral septa were visualized and two others were suspected. Thus, in only $50 \%$ of cases were neuroimaging studies helpful in predicting the presence of ventral cord tethering.

A prevertebral neurenteric cyst was revealed by CT myelography in a case of low-cervical ventral septum that was traceable to an intestinal band. Counting all the studies in which CT myelography successfully visualized the lesions, either intestinal malformations or dermal sinus tracts were present in all but two cases.

Only four cases of an anterior bifid vertebra were seen in these 11 patients, including the one found at autopsy.

\section{Predictors of Ventral Tethering}

Various anatomical, clinical, and radiographical parameters are compared between the group of type II SCM with ventral septa (11 cases) and the group with purely dorsal septa or no septum (41 cases, Table 1) in an attempt to determine factors that might predict the presence of a ventral septum before undertaking surgical exploration.

The only absolutely reliable predictor seems to be the presence of an associated intestinal malrotation or dupli- cation (diverticulum) or the finding of a dermal sinus tract directly continuous with the median septum. In each of these cases, a ventral septum was found, always connected to remnants or derivatives of the original endomesenchymal tract.

A higher percentage of patients with a ventral septum were symptomatic compared to patients with a dorsal septum. Myelopathy was slightly more common in the group with ventral tethering, perhaps because of the proximity of the septum to the corticospinal tracts.

Computerized tomography myelography predicted ventral tethering in approximately half of the patients studied, as compared with a predictability rate of $38 \%$ in patients with a purely dorsal septum. Magnetic resonance imaging is utterly ineffective in detecting any fibrous septum in type II SCMs, ventral or dorsal.

The location of the septum, split length, the preponderance of myelomeningocele manqués and thickened fila, anterior bifid vertebrae, and hemimyelomeningoceles, all have no predictive value for the presence of ventral cord tethering in type II SCM.

\section{Ventral Cord Tethering in Type I SCM}

I have encountered two settings in which the hemicords were tethered anteriorly in type I SCM after surgical treatment. Three patients were sent to us with recurrent symptoms after they had undergone resection of the bone septum and the dural sleeve. In two patients, the bone spike was inadequately resected; there was a sizeable stump not flushed with the ventral dura, and the hemicords were caught at the reunion "crotch." In the third patient, the dural sleeve was inadequately resected. 


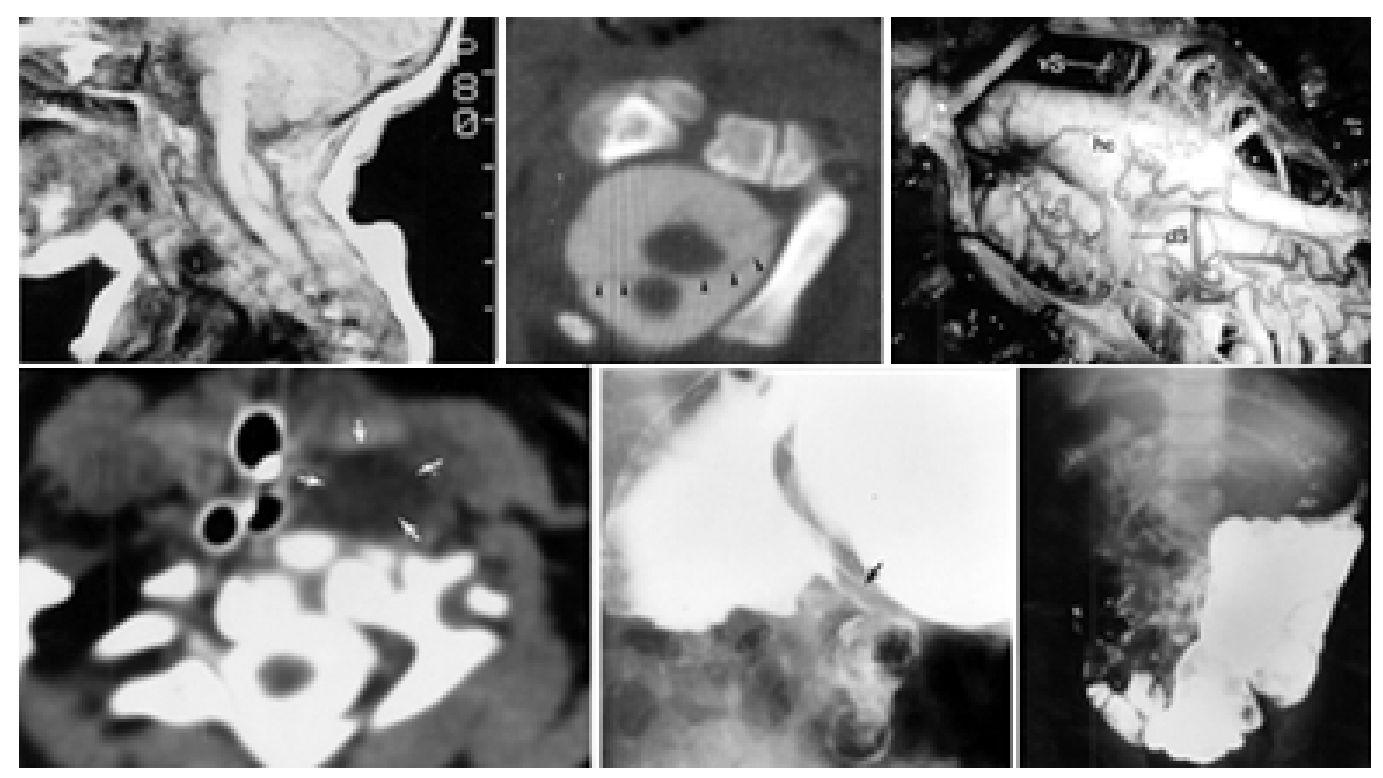

Fig. 5. Complete dorsoventral fibrous septum in type II SCM associated with intestinal malrotation. Upper Left: Sagittal MR imaging revealing the anteriorposterior arrangement of the hemicords. Upper Center: CT myelogram obtained at C-3 demonstrating the oblique fibrous septum (arrowheads) and the two hemicords. Note the bifid VB. Upper Right: At surgery, one hemicord (hc) is seen to be roughly piggy-backed on the other. The dorsal fibrous septum (dS) is obliquely tethering the hemicords to the dorsal dura, and the ventral septum (vS) is tethered to the ventral dura. Lower Left: A CT myelogram obtained at C-7 revealing the prevertebral neurenteric cyst (arrows) related closely to the bifid VB. Lower Center: Upper gastrointestinal series showing duodenal obstruction at the duodenal jejunal junction caused by a crossing fibrous band attached to the fourth part of the duodenum (arrow) and the cecum at one end, and the retroperitoneum at the other. Lower Right: The terminal ileum inserts into the ascending colon from the right side, and the large bowels are all on the left side of the abdomen, indicating complete intestinal malrotation.

The other setting of "acquired" ventral tethering in type I SCM was observed in cases (two patients) in which the hemicords were adherent to the suture line used to repair the anterior dural defect left by resection of the median dural sleeve of a type I bone spur.

\section{DISCUSSION}

\section{Embryogenesis of Ventral Tethering in SCM}

In 1992, we proposed that the basic error of all SCM types occurs very early in the development of the embryo. During the transformation of the bilaminar to the trilaminar embryo in early gastrulation, one of the cardinal events is formation of the notochord. Spratt ${ }^{32}$ demonstrated years ago that the notochord becomes elongated by the addition of new cells to the caudal end of a short head process that points toward the cephalic end of the embryo. The notochord therefore grows rearward, as it were, along with backward expansion of the lateral wings of the embryonic plate upon gradual establishment of the three germ layers of ectoderm, mesoderm, and endoderm. Backward elongation of the notochord is also concomitant with shrinking of the primitive streak, as the stem cells from the latter structure are being "exhausted" to form the new germ layers.

The prospective notochord cells are derived from the Hensen node. They stream past the primitive pit to wedge between the ectoderm and the endoderm in the midline. These prospective notochordal cells, stemming equinumerically from each bank of the primitive knot (Hensen node), must in some way achieve midline integration as they line up at the caudal end of the elongating notochord, to become a solid cord. This midline integration most likely involves a cell adhesion molecule, probably fibronectin, which is known to exist in the early epochs. ${ }^{17}$

The basic error in all SCMs is very possibly a failure of midline integration of the newly forming notochordal

TABLE 1

Comparison between type II SCM with and without ventral tethering*

\begin{tabular}{lcc}
\hline \hline \multicolumn{1}{c}{ Variable } & $\begin{array}{c}\text { Type II SCM } \\
\text { W/ Ventral } \\
\text { Tethering (\%) }\end{array}$ & $\begin{array}{c}\text { Type II SCM } \\
\text { W/O Ventral } \\
\text { Tethering (\%) }\end{array}$ \\
\hline no. of patients & 11 & 41 \\
location of septum & 2 C, 3 T, 6 LS & 6 C, 8 T, 27 LS \\
mean split length & & \\
$\quad$ in vertebral level) & 1.4 & 1.8 \\
myelomeningocele manqué & $6(55)$ & $20(49)$ \\
thickened filum & $9(82)$ & $33(80)$ \\
hemimyelomeningocele & $2(18)$ & $3(7)$ \\
anterior bifid vertebra & $4(36)$ & $12(29)$ \\
symptomatic/progressive deficit & $10(91)$ & $14(34)$ \\
myelopathy & $6(55)$ & 0 \\
intestinal malformation & 3 & 0 \\
dermoid sinus tract & 2 & 0 of 33 \\
septum shown on MR imaging & 0 of 9 & $14(38) 37$ \\
septum shown on CTM & $5(50) 10$ & \\
\hline
\end{tabular}

$* \mathrm{C}=$ cervical $\mathrm{CTM}=\mathrm{CT}$ myelogram $; \mathrm{LS}=$ lumbosacral $\mathrm{T}=$ thoracic. 


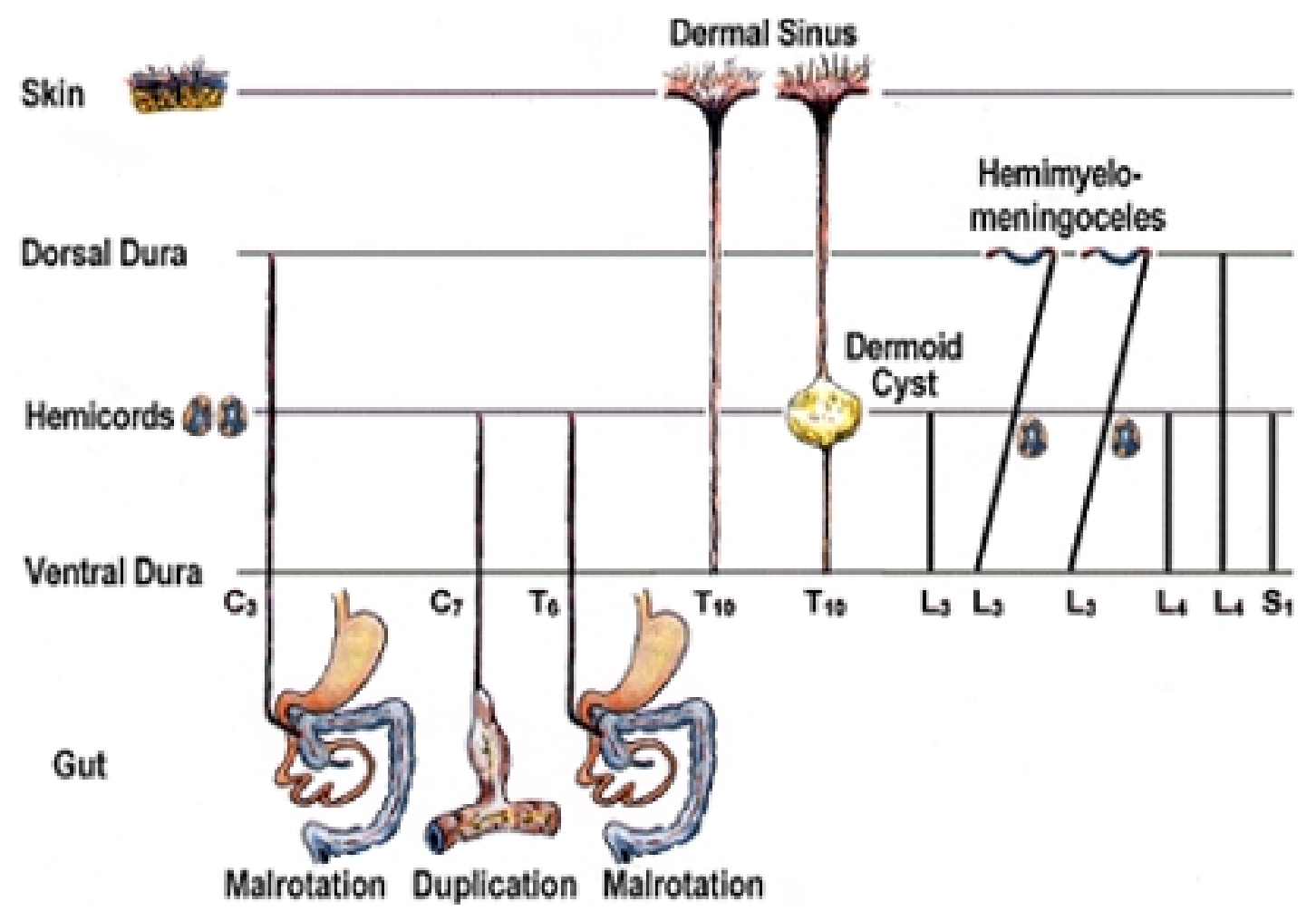

Fig. 6. Schematic drawing showing the four categories of ventral septa and their related malformations, laid out from left to right for cervical to sacral level and from top to bottom for dorsal to ventral side of the embryo. Category 1: intradural ventral septum (L-3, L-4; S-1); Category 2: intradural dorsoventral complete septum (L-2, L-3; L-4); Category 3: intradural dorsoventral complete septum continuous with dermal sinus tract (T-10, T-10); and Category 4: ventral or complete septum attached to primitive gut (C-3, C-7, T-6).

cells. ${ }^{7}$ We must conjecture on the mechanism of this failure, but it may be due to a defective cell adhesion molecule, or a mistiming, or even misplacement of the normal molecule. If this failure of midline integration occurs only for a brief moment in time, in a square pulse fashion, the result will be a small midline area of persistent adhesion between the ectoderm and the endoderm, flanked by two heminotochords.

This dorsoventral adhesion tract, both topographically and figuratively central to our theme of SCMs, must then contain ectoderm and endoderm in queue. It is also logical to expect the ubiquitous mesenchymal cells to form close alliance with this tract, themselves being part of that highly migrant, pleurigenic cell pool that ultimately supplies all the mesodermal derivatives of the embryo. This adhesion can now properly be called the endomesenchymal tract, or more precisely, the ectoendomesenchymal tract, in honor of its constituent cell populations. Slightly later, around postovulation Day 30, a fourth group of progenitor cells derived from the primitive meninx, newly gelating between the notochord and the neural plate, ${ }^{20}$ becomes involved with the endomesenchymal tract. ${ }^{24,25}$

After formation of the endomesenchymal tract, the final shaping of the mature malformation around it depends on four variable developmental circumstances operating simultaneously: 1) growth of the progenies of the four cellular components; 2) persistence of the endomesenchymal tract; 3) anomalous interaction between the hemineural plates and the heminotochords during neurulation; and 4) midline healing of the split neural plates and notochord. ${ }^{25}$

Whether primitive meninx cells, with their bivalent ability to form bone and pachymeninx, are spatiotemporally available to be incorporated into the endomesenchymal tract will determine if the hemicords are bisected by a bone spur in between two separate dural sacs (type I SCM) or contained within a common dural sac without a median bone septum (type II SCM). ${ }^{25}$ The mesenchyme

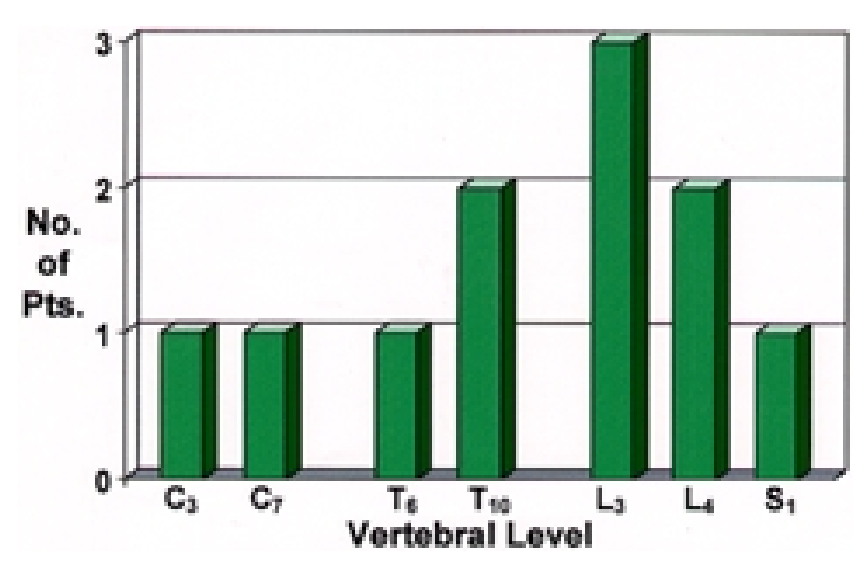

Fig. 7. Bar graph indicating the location of the ventral fibrous septa. Pts $=$ patients. 


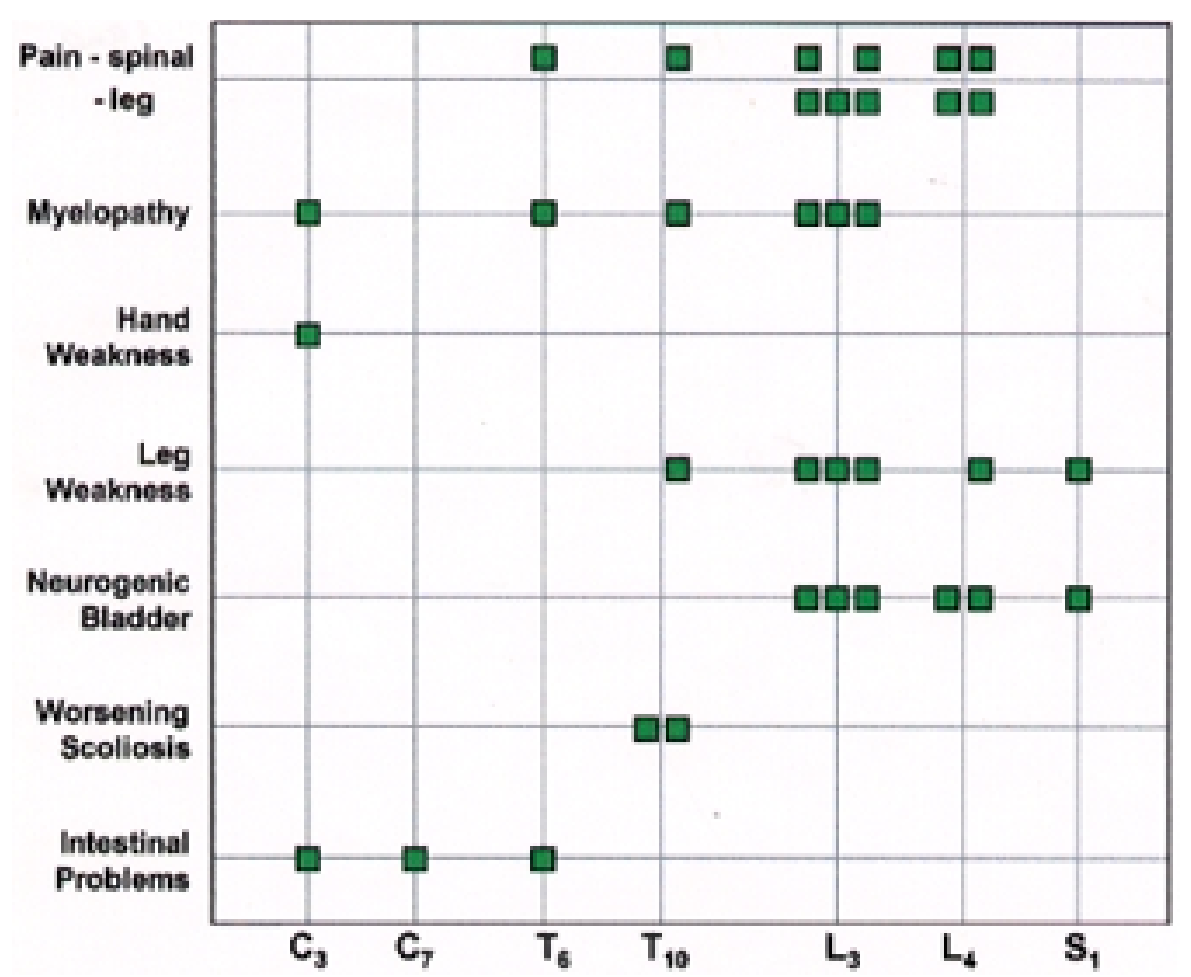

Fig. 8. Neurological and intestinal problems correlated with the level of the ventral tethering.

within a type II tract may form strong fibrous septa and/or bands that tether the hemicords to the adjacent dura. Insofar as the original endomesenchymal tract bisects the entire neural canal, the surprising feature of type II SCM is not so much why ventral septa exist, but why they exist only in a relatively small number as compared with dorsal septa. One explanation may be a different induction milieu between the ventral and dorsal surfaces of the neural plate, but given that mesenchymal induction occurs practically everywhere in the embryo, the real explanation is wanting. Given the pluripotential nature of mesenchymal cells, both dorsal and ventral fibrous septa are frequently associated or even embedded with large blood vessels, fat, and muscle. If neural crests cells are also entrapped by the endomesenchymal tract, paramedian dorsal nerve roots (being central processes of dorsal ganglion cells) and ganglia cells will form between the dorsal medial aspect of the hemicords and the dorsal dura along side the fibrous bands and vessels, giving rise to myelomeningocele manqués. The absence of neural crest cells ventral to the neural plate explains why no myelomeningocele manqués are ever involved in ventral tethering.

Unlike mesenchymal derivatives that appear to exist in some form or other in every case of SCM, ectodermal stem cells only rarely develop into dermal sinus tract or dermoid cysts. ${ }^{22}$ Even rarer is the association of neurenteric cysts with SCM, perhaps because of the fastidious nature of endodermal ontogeny in unscheduled locations such as deep within the median cleft of a SCM, beyond reach of the usual inducer molecules.

In most cases of SCM, only the intradural portion of the endomesenchymal tract persists as the mesodermal derivative(s) of the median cleft. In the exceedingly rare case in which the entire endomesenchymal tract remains as a patent primitive canal connecting the yolk sac with the amniotic sac, part of the intestine may herniate through this canal and appear on the dorsum of the embryo as an in-

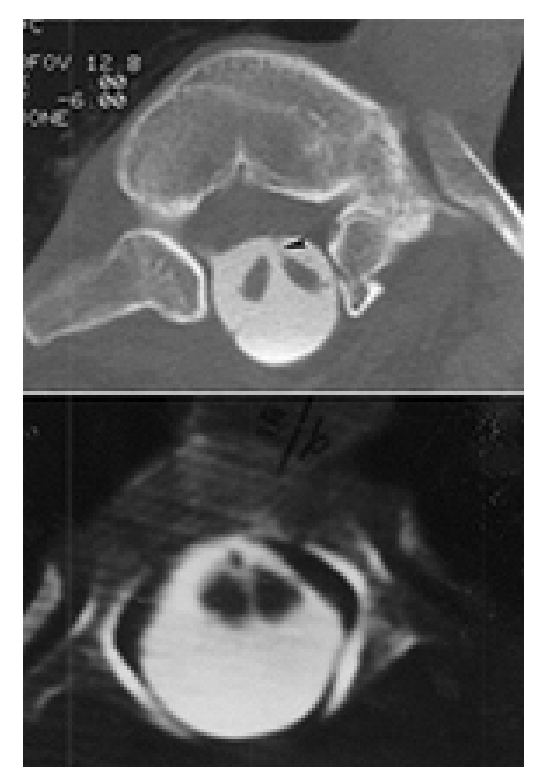

Fig. 9. Computerized tomography myelograms demonstrating two type II SCMs suggestive of but not definitively showing a ventral septum. Upper: A ventral structure (arrowhead) is attached to one hemicord, suggesting ventral tethering. Lower: An extra "shadow" is shown in the ventral midline but not a complete septum. 


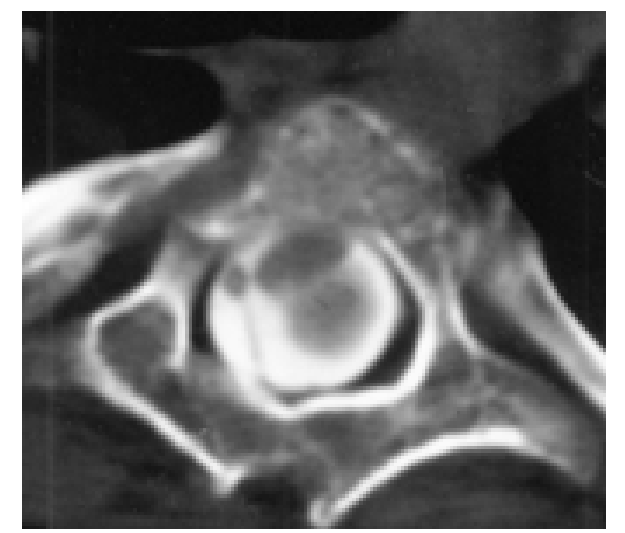

Fig. 10. A type II SCM with intradural dermoid cyst demonstrated on CT myelography (case depicted in Fig. 3), showing very clearly a dorsal septum attached to the smaller hemicord, but no ventral septum is delineated.

testinal fistula. ${ }^{5}$ In the less rare situation in which only the ventral link with the yolk sac is retained, the pulling effect of this tract could result in an intestinal diverticulum (duplication) pointing toward the prevertebral (prenotochordal) portion of the original endomesenchymal tract. ${ }^{2,3,10,18,19}$, ${ }^{24,25,36}$ Alternately the tract may prevent normal midgut rotation to cause intestinal malrotation..$^{2,14,18,25,33}$ If only the dorsal endomesenchymal tract remains, the ectodermal elements may form a dermal sinus opening and tract continuous with the median septum. It is probably not coincidence that whenever a retained ventral or dorsal extradural tract exists, the intradural fibrous septum is both dorsal and ventral as part of an unbroken continuum. One assumes the factors that permit retention of the extradural endomesenchymal tract also promote condensation of the ventral intradural sheath.

Normal neurulation begins with formation of the floor plate, which in turn is dependent solely on inductive influences of the adjacent notochord. ${ }^{29-31,35,37}$ The notochords of Xenopus ${ }^{11}$ and humans ${ }^{15}$ are both known to express the sonic hedgehog gene, which induces the floor plate to respond to signals from the notochord. ${ }^{11}$ Such interactions are spatially and directionally programmed and may be profoundly disturbed if the hemineural plate is too far removed from its heminotochord partner, as would be the case if the hemineural plates are somehow dorsally dislocated during neurulation. ${ }^{27}$ The actual dorsal bending of the neural plates is normally impelled by balanced forces provided by the enlarging paraxial mesoderms on each side and by longitudinal stretching of the elongating cutaneous ectoderm. ${ }^{28,30}$ Movements of a hemineural plate by unopposed unilateral forces must occasionally place it at an untenable distance from the heminotochord. The result could conceivably be a full-fledged myelomeningocele with paired unneurulated hemiplacodes, or a hemimyelomeningocele with one neurulated and one unneurulated placode. ${ }^{23}$ Both cases of hemimyelomeningocele in this series had complete dorsoventral fibrous septa that were oblique, suggesting that an earlier dorsoventral rotation of the twin hemineural plates had brought the unneurulated mate dorsally far away from the source of the inducer gene.

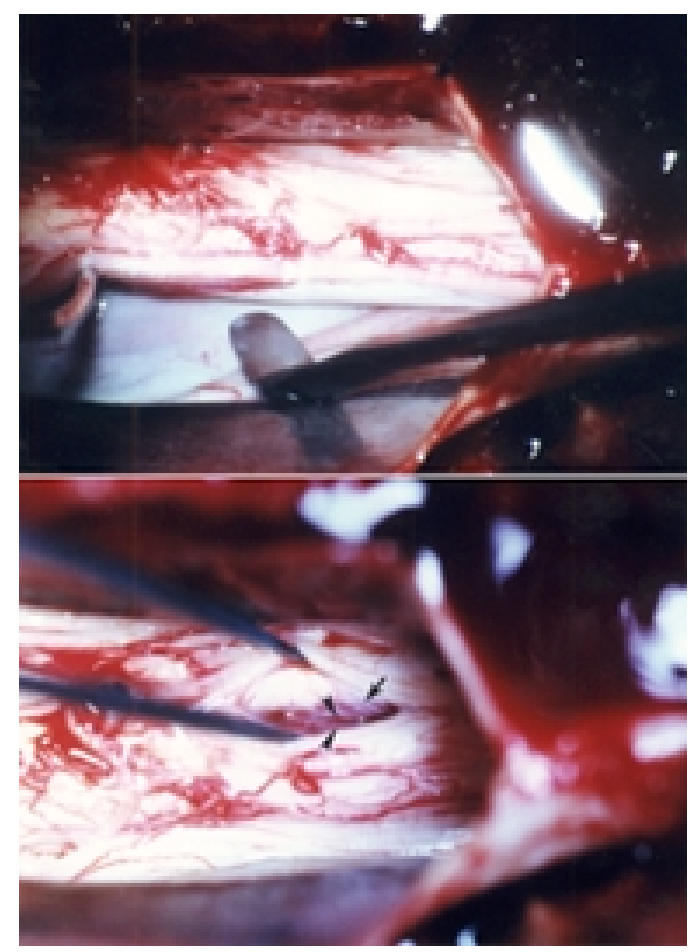

Fig. 11. Upper: Ventral exploration by gently lifting each hemicord by turn. The ventral intradural space is visualized and then "palpated" by an angled spatula microdissector. No ventral band is seen in this case. Lower: Transcleft exploration. Note the duplicated anterior spinal arteries (arrowheads) half hidden by the hemicord, and the remnants of the ventral septum (arrow) attachment to the hemicord.

Finally, the capacity of the neural tube and notochord to heal in the middle will determine whether there are two widely sundered hemicords or merely a cleft cord as well as whether the VB is bifid or just misshapen. Our modest numbers suggest that the factors enhancing midline healing are not influenced by the presence of a ventral mesenchymal septum.

\section{Clinical Significance of Ventral Septa}

It is surprising that ventral tethering in type II SCM has not received any attention in recent literature. Ersahin, et al., ${ }^{9}$ never specifically mentioned ventral septa in their 18 patients with type II SCM, although they described in detail two unusual cases of type II SCM in which the hemicords were separated by a through-and-through sagittal fibrous septum that was attached ventrally to a bony excrescence arising from the VB. Birch and McCormick ${ }^{4}$ reported one case of high-cervical type II split cord in which a ventral neurenteric cyst and ventral septum were present. The seven patients with cervical type II lesions reported by David, et al., ${ }^{6}$ never underwent surgical exploration. In at least 20 other operative cases of type II SCM described in the literature, there is no specific mention of either ventral septum or ventral exploration. ${ }^{1,13,21,26}$

Based on a few simple statistical results from this study, type II SCM should be recognized as a culpable lesion in general, and the ventral septum as a particularly troublesome subtype of tethering (Table 1). Sixty-seven 
percent of all patients with type II lesions were either symptomatic or suffering from progressive neurological deterioration. Withinn the type II family of lesions, $61 \%$ of patients with purely dorsal septa experienced neurological progression, as compared with $91 \%$ of those with some type of ventral attachment. If the one autopsy case in the latter subgroup is excluded, every patient with ventral tethering experienced neurological deterioration prior to diagnosis.

Although they did not reach statistical significance, our data also show that in $55 \%$ of those with ventral tethering, myelopathic signs were shown as compared with $34 \%$ in those with purely dorsal tethering. This finding suggests ventral tethering of the hemicords is in some way more deleterious to the corticospinal tracts than pure dorsal tethering.

Thus, the surgical exploration of all type II lesions should no longer be controversial regardless of whether neuroimaging studies reveal definite septa. I would further endorse a thorough exploration of the ventral surface of each type II split cord to rule out a ventral tethering band. Some authors may argue that the associated thickened filum in many cases of SCM and not the fibrous septum itself is responsible for the conus medullaris injury, but there are now ample examples of recurrent tethering observed after simple division of the filum (without releasing the hemicords), as well as cervical and thoracic SCMs without thickened filum, to implicate the median fibrous bands as the real pathogenetic culprit.

\section{Techniques of Surgery}

Ventral exploration of a type II SCM should be conducted by gently lifting and rotating one hemicord away from the midline plane (Fig. 11 upper). A better angle of vision is obtained by making a wide bone exposure from side to side. This is usually possible because the spinal canal at the split cord level is often wider than the adjacent segments. If a dorsal fibrous septum or large myelomeningocele manqués are present, they should first be cut to ensure a maximum degree of freedom before manipulation of the hemicords.

Exploration through the median cleft is usually not advised because there are often tight adhesion bands binding the hemicords deep within the cleft. Navigating through these bands would additionally confound what already amounts to an end-on view of the ventral septum. The operator may find himself entangled within the two sleeves of a septum while attempting to cut the sleeves in a plane perpendicular to his line of vision, at the same time struggling to avoid injuring the hemicords. The anterior spinal artery, which may be duplicated, is partially hidden by the hemicords and, thus, is vulnerable to injury by transcleft dissection (Fig. 12 lower). Finally, the use of electrocautery deep within the cleft may inflict thermal injury to the hemicords.

Only one situation justifies the use of the transcleft approach: the removal of a neurenteric cyst or teratoma buried in the folds of the hemicords. ${ }^{8,12,16,34}$

\section{Predictors of Ventral Tethering}

The present neuroimaging results revalidate our earlier claim that MR imaging is wholly unsuccessful, and CT myelography only partially successful, in revealing median fibrous septa in type II SCM. ${ }^{23}$ The presence of a ventral septum was conclusively predicted in $30 \%$ and tentatively predicted in $20 \%$ based on CT myelography studies. If we were to adhere to some authors' rather nihilistic philosophy of only treating septa that were demonstrated radiographically, ${ }^{5,13}$ then $50 \%$ of our patients with tethered hemicords would have been ignored. Our analysis also shows that the location of septum, split length, neurological presentation, and the presence of myelomeningocele manqué and thickened filum have no correlational value for diagnosing ventral tethering (Table 1).

Are there, then, any preoperative predictors of ventral tethering that could spare us the efforts and risks of manipulating at least some hemicords? Certainly, if the CT myelography demonstrates a definite septum or even the ghost of one, the yield should be high. Otherwise, the only strong associations with ventral septum are persistent banding with the primitive gut and dermal ectodermal tracts opened to the skin. The number of such cases in our series is small, and the literature is not helpful (Ersahin's ${ }^{9}$ two well-illustrated cases of ventral septum both had dermal sinus tracts). It remains to be seen whether a larger number of such cases would confirm these predictions. In the meantime, we are left with the hypothesis that embryological events acting to preserve the original endomesenchymal linkage to the yolk sac and skin also seem inclined to materialize the intradural tract. In this regard, one would expect anterior bifid vertebrae to be more commonly found with ventral septa, but our numbers fail to verify this.

To put into perspective, even if we combine the positive findings demonstrated on CT myelography studies with cases of known intestinal malformations and dermal sinus tracts (several cases overlap), only seven patients could be considered high risk for ventral tethering. Almost $40 \%$ with ventral septa would have escaped detection if surgical exploration had not been done.

\section{Acquired Ventral Tethering in Type I SCM}

The development of acquired ventral tethering due to inadequate resection of a type I bone spur or dural sleeve is self-explanatory. The other kind of acquired ventral tethering requires elaboration and clarification because it is intuitive for neurosurgeons to close a dural opening by suturing. It took two negative experiences to convince me that an exception must be made for the case of the ventral dural defect left after removal of a median dural sleeve. In a very early case in the series, I foolishly used a rightangled flat dissector to explore the ventral epidural space via the ventral dural opening that is invariably present after the dural sleeve is cut flush with the VB. This resulted in the development of a pseudomeningocele from leakage of CSF through the ventral rent and then round the anterior dural sac to the back. In repairing this pseudomeningocele, I sutured the anterior dural rent with prolene sutures. Thinking I was learning from my own mistake, I sutured the anterior dural defect in the next case of type I SCM even though there was no CSF leak. Both patients promptly developed recurrent symptoms due to ventral adherence of the hemicords to the suture line. I now realize that as long as the natural adhesions in the ventral epidural space are not disturbed, CSF leakage never occurs, 
and the anterior dural opening can be left unsutured. Since then, no other acquired ventral tethering or pseudomeningocele has been observed.

\section{CONCLUSIONS}

1) Twenty-one percent of patients with a type II SCM harbor a type of ventral tethering band. 2) The incidence of neurological deterioration, especially myelopathy, is slightly higher in cases of ventral tethering than those of pure dorsal tethering. 3) Computerized tomography myelography has less than $50 \%$ accuracy in revealing the ventral band. The only other predictor of ventral tethering seems to be a coexisting intestinal diverticulum or malrotation, or a dermal sinus tract. Even so, $40 \%$ of ventral tethering in type II SCM remains elusive. 4) I recommend thorough exploration of the ventral aspects of the hemicords during surgery for type II SCM. 5) The ventral dural defect resulting from resection of a type I bone spur and dural sleeve should be left open to avoid iatrogenic ventral tethering.

\section{References}

1. Andar UB, Harkness WF, Hayward RD: Split cord malformations of the lumbar region. A model for the neurosurgical management of all types of 'occult' spinal dysraphism? Pediatr Neurosurg 26: 17-24, 1997

2. Beardmore HE, Wiglesworth FW: Vertebral anomalies and alimentary duplications. Pediatr Clin North Am 5:457-474, 1958

3. Bentley JFR, Smith JR: Developmental posterior enteric remnants and spinal malformations. Arch Dis Child 35:76-86, 1960

4. Birch BD, McCormick P: High cervical split cord malformation and neurenteric cyst associated with congenital mirror movements: case report. Neurosurgery 38:813-816, 1996

5. Bremer JL: Dorsal intestinal fistula; accessory neurenteric canal; diastomyelia. Arch Pathol 54:53-54, 1952

6. David KM, Copp AJ, Stevens JM, et al: Split cervical spinal cord with Klippel-Feil syndrome: seven cases. Brain 119: 1859-1872, 1996

7. Dias MS, Pang D: Split cord malformations. Neurosurg Clin North Am 6:339-358, 1995

8. Ersahin Y, Demirtas E, Mutluer S, et al: Split cord malformations: report of three unusual cases. Pediatr Neurosurg 24: 155-159, 1996

9. Ersahin Y, Mutluer S, Kocaman S, et al: Split spinal cord malformations in children. J Neurosurg 88:57-65, 1998

10. Fallon M, Gordon ARG, Lendrum ACZ: Mediastinal cysts of fore-gut origin associated with vertebral abnormalities. Br J Surg 41:520-533, 1954

11. Flitman HP, Stanek J, Hsu HW, et al: Anomalous ependyma inducing split cord and meningomyelocele? J Child Neurol 14: 473-177, 1999

12. Hader WJ, Steinbok P, Poskitt K, et al: Intramedullary spinal teratoma and diastematomyelia. Case report and review of the literature. Pediatr Neurosurg 30:140-145, 1999

13. Iskandar BJ, Fulmer BB, Hadley MN, et al: Congenital tethered spinal cord syndrome in adults. J Neurosurg 88:958-961, 1998

14. James CCM, Lassman LP: Spinal dysraphism: the diagnosis and treatment of progressive lesions in spina bifida occulta. $\mathbf{J}$ Bone Joint Surg (Br) 44:828-840, 1962

15. Kirillova I, Novikova I, Auge J, et al: Expression of the sonic hedgehog gene in human embryos with neural tube defects. Teratology 61:347-354, 2000
16. Koen JL, McLendon RE, George TM: Intradural spinal teratoma: evidence for a dysembryogenic origin. Report of four cases. J Neurosurg 89:844-851, 1998

17. Krolo M, Vilovic K, Sapunar D, et al: Fibronectin expression in the developing human spinal cord, nerves, and ganglia. Croatian Med J 39:386-391, 1998

18. McLetchie NGB, Purves JK, Saunders RL: The genesis of gastric and certain intestinal diverticula and enterogenous cysts. Surg Gynecol Obstet 99:135-141, 1954

19. Nathan MT: Cysts and duplications of neurenteric origin. Pediatrics 23:476-484, 1959

20. O'Rahilly R, Meyer DB: The timing and sequence of events in the development of the human vertebral column during the embryonic period proper. Anat Embryol 157:157-176, 1979

21. Ozer H, Yuccer N: Myelomeningocele, dermal sinus tract, split cord malformation associates with extradural teratoma in a 30month-old girl. Acta Neuroshir 141:1123-1124, 1999

22. Pang D: Split cord malformation, in Pang D (ed): Disorders of the Pediatric Spine. New York: Raven Press, 1995, pp 203-251

23. Pang D: Split cord malformation: Part II: Clinical syndrome Neurosurgery 31:481-500, 1992

24. Pang D: Surgical management of split cord malformations, in Wilkins RH, Rengachary SS (eds): Neurosurgical Operative Atlas. Baltimore: Williams \& Wilkins, 1993, Vol 3, pp 135-149

25. Pang D, Dias MS, Ahab-Barmada M: Split cord malformation: Part I: A unified theory of embryogenesis for double spinal cord malformations. Neurosurgery 31:451-480, 1992

26. Proctor MR, Bauer SB, Scott RM: The effect of surgery for split spinal cord malformation on neurologic and urologic function. Pediatr Neurosurg 32:13-19, 2000

27. Saraga-Babic, M, Stefanovic V, Wartiovaara J, et al: Spinal cord-notochord relationship in normal human embryos and in a human embryo with double spinal cord. Acta Neuropathol 86: 509-514, 1993

28. Schoenwolf GC: Microsurgical analyses of avian neurulation: separation of medial and lateral itssues. J Comp Neurol 276: 498-507, 1988

29. Schoenwolf GC, Desmond ME: Descriptive studies of occlusion and reopening of the spinal canal of the early chick embryo. Anat Rec 209:251-263, 1984

30. Schoenwolf GC, Franks MV: Quantitative analyses of changes in cell shapes during bending of the avian neural plate. Dev Biol 105:257-272, 1984

31. Smith JL, Schoenwolf GC: Notochordal induction of cell wedging in the chick neural plate and its role in neural tube formation. J Exp Zool 250:49-62, 1989

32. Spratt NT: Regression and shortening of the primitive streak in the explanted chick blastoderm. J Exp Zool 104:69-100, 1947

33. Till K: Spinal dysraphism: a study of congenital malformations of the back. Dev Med Child Neurol 10:471-477, 1968

34. Ugarte N, Gonzalez-Crussi F, Sotelo-Avila C: Diastematomyelia associated with teratomas. Report of two cases. J Neurosurg 53:720-725, 1980

35. van Straaten HWM, Hekking JWM, Thors F, et al: Induction of an additional floor plate in the neural tube. Acta Morphol Neerl Scand 23:91-97, 1985

36. Veeneklass GMH: Pathogenesis of intrathoracic gastrogenic cysts. Am J Dis Child 83:500-507, 1952

37. Youn BW, Malacinski GM: Axial structure development in ultraviolet-irradiated (notochord-defective) amphibian embryos. Dev Biol 83:339-352, 1981

Manuscript received December 4, 2000.

Accepted in final form December 21, 2000.

Address reprint requests to: Dachling Pang, M.D., F.R.C.S.(C), F.A.C.S., Department of Pediatric Neurosurgery, Kaiser Permanente Hospitals, 2025 Morse Avenue, Sacramento, California 95825. 